\title{
PENALIZED NONPARAMETRIC DRIFT ESTIMATION FOR A CONTINUOUSLY OBSERVED ONE-DIMENSIONAL DIFFUSION PROCESS
}

\author{
Eva LÖCherbach ${ }^{1}$, Dasha Loukianova ${ }^{2}$ and Oleg Loukianov ${ }^{3}$
}

\begin{abstract}
Let $X$ be a one dimensional positive recurrent diffusion continuously observed on $[0, t]$. We consider a non parametric estimator of the drift function on a given interval. Our estimator, obtained using a penalized least square approach, belongs to a finite dimensional functional space, whose dimension is selected according to the data. The non-asymptotic risk-bound reaches the minimax optimal rate of convergence when $t \rightarrow \infty$. The main point of our work is that we do not suppose the process to be in stationary regime neither to be exponentially $\beta$-mixing. This is possible thanks to the use of a new polynomial inequality in the ergodic theorem [E. Löcherbach, D. Loukianova and O. Loukianov, Ann. Inst. H. Poincaré Probab. Statist. 47 (2011) 425-449].
\end{abstract}

Mathematics Subject Classification. 60F99, 60J35, 60J55, 60J60, 62G99, 62M05.

Received March 25, 2009. Revised October 6, 2009.

\section{INTRODUCTION}

Let $X_{t}$ be a one-dimensional diffusion process given by

$$
\mathrm{d} X_{t}=b\left(X_{t}\right) \mathrm{d} t+\sigma\left(X_{t}\right) \mathrm{d} W_{t}, \quad X_{0}=x,
$$

where $W$ is a standard Brownian motion. Assuming that the process is positive recurrent but not necessarily in the stationary regime (i.e. not starting from the invariant measure) and not necessarily exponentially $\beta$-mixing, we want to estimate the unknown drift function $b$ on a fixed interval $K$ from observations of $X$ during the time interval $[0, t]$, for fixed $t$. We do not require any knowledge about smoothness of the drift function: $b$ is not supposed to belong to some known Besov or Sobolev ball. Hence we aim at studying nonparametric adaptive estimators for the unknown drift $b$.

\footnotetext{
Keywords and phrases. Diffusion process, adaptive estimation, regeneration method, mean square estimator, model selection, deviation inequalities.

${ }^{1}$ Centre de Mathématiques, Faculté de Sciences et Technologie, Université Paris-Est Val-de-Marne, 61 avenue du Général de Gaulle, 94010 Créteil, France; locherbach@univ-paris12.fr

2 Département de Mathématiques, Université d'Evry-Val d'Essonne, Bd François Mitterrand, 91025 Evry, France;

dasha.loukianova@univ-evry.fr

${ }^{3}$ Département Informatique, IUT de Fontainebleau, Université Paris-Est, route Hurtault, 77300 Fontainebleau, France;

oleg@iut-fbleau.fr
} 
Nonparametric estimation in continuous time of the drift coefficient of diffusion processes has been widely studied over the last decades. To mention just a few, let us cite Banon [1], Prakasa Rao [18], Pham [17], Galtchouk and Pergamenschikov [9], Dalalyan and Kutoyants [7], Delattre, Hoffmann and Kessler [8], Loukianova and Loukianov [14], Löcherbach and Loukianova [15] and the extensive book of Kutoyants [11].

The adaptive estimation for the drift at a fixed point has been studied by Spokoiny [20], who uses Lepskii's method (see [12]) in order to construct an adaptive procedure. Dalalyan [6], uses kernel-type estimators and considers a weighted $L^{2}$-risk, where the weight is given by the invariant density. He has to work under quite strong ergodicity assumptions.

Our aim in this paper is twofold. Firstly, we aim at introducing a nonparametric estimation procedure based on model selection. Our estimator is obtained by minimizing a contrast function within a fixed finite-dimensional linear sub-space of $L^{2}(K, \mathrm{~d} x)$ - quite in the spirit of mean square estimation and following ideas presented by Comte et al. [5], for discretely observed diffusions. These finite-dimensional sub-spaces include spaces such as piecewise polynomials or compactly supported wavelets. The risk we consider for a given estimator $\hat{b}$ of $b$ is the expectation of an empirical $L^{2}$-norm defined by

$$
\mathbb{E}_{x}\|\hat{b}-b\|_{t}^{2}, \text { where }\|\hat{b}-b\|_{t}^{2}=\frac{1}{t} \int_{0}^{t}(\hat{b}-b)^{2}\left(X_{s}\right) \mathrm{d} s .
$$

The dimension of the space is chosen by a data-driven method using a penalization.

Secondly, we aim at working under the less restrictive assumptions on the ergodicity properties of the process that seem to be possible. We do not impose the diffusion to be exponentially $\beta$-mixing and do not assume the existence of exponential moments for the invariant measure, though we do have to impose the existence of a certain number of moments. Finally, note that we do not work in the stationary regime: the process starts from a fixed point $x \in K$, and is not yet in equilibrium. Note also that our approach is non-asymptotic in time. But we have to suppose that $t \geq t_{0}$ for some fixed explicitly given time horizon $t_{0}$ that is needed for theoretical reasons and defined precisely later in the text (see Prop. 3.4). A main ingredient of the proofs is a new polynomial inequality ensuring that empirical norm and theoretical $L^{2}$-norm are not too far away. This inequality is given in Loukianova et al. [16].

The paper is organized as follows. In Section 2 we describe our framework and give the main results: in Section 2.1 we give precise assumptions on the diffusion model, explain these assumptions and give some examples for models satisfying them. In Section 2.2 we introduce both the non-adaptive and adaptive estimator, Section 2.3 gives assumptions on the approximation spaces and Section 2.4 provides some examples of approximation spaces verifying these assumptions. The main results (rate of convergence of estimators) are given in Section 2.5. Section 3 presents probabilistic tools and auxiliary results necessary for the proof of the main results. Section 4 is devoted to the proofs of the main results: Section 4.1 deals with non-adaptive and Section 4.2 with adaptive drift estimation. Finally, Section 5 is an appendix, where we give the proof of one technical result (Lem. 4.2).

\section{Framework, ASSUmptions AND MAIN RESUlts}

\subsection{Assumptions on the diffusion}

Let $X_{t}$ be a one-dimensional diffusion process given by

$$
\mathrm{d} X_{t}=b\left(X_{t}\right) \mathrm{d} t+\sigma\left(X_{t}\right) \mathrm{d} W_{t}, \quad X_{0}=x .
$$

We would like to estimate the drift function $b$ on a fixed interval $K$, say $K=[0,1]$. To insure the existence and the unicity of a strong non exploding solution of (2.1) we suppose

\section{Assumption 2.1.}

1. $b$ and $\sigma$ are locally Lipschitz and $b$ is at most of linear growth. 
2. There exist $0<\sigma_{0}^{2} \leq \sigma_{1}^{2}<\infty$ such that for all $x, \sigma_{0}^{2} \leq \sigma^{2}(x) \leq \sigma_{1}^{2}$.

A more particular assumption is needed for the drift function to guarantee some "speed" of ergodicity of $X$.

\section{Assumption 2.2.}

1. There are two known constants $M_{0}$ and $b_{0}$ such that $K \subset\left[-M_{0}, M_{0}\right]$ and for all $x$ with $|x| \leq M_{0}$, $|b(x)| \leq b_{0}$.

2. We suppose that there is a positive constant $\gamma$ such that for all $x$ with $|x| \geq M_{0}$,

$$
x b(x) \leq-\gamma .
$$

3. The constant $\gamma$ satisfy $2 \gamma>31 \sigma_{1}^{2}$.

To clarify the meaning of Assumptions 2.2 let us recall some well-known facts about linear diffusions. We refer the reader to the book of Revuz and Yor [19]. The scale density of $X$ is given by

$$
s(x)=\exp \left(-2 \int_{0}^{x} \frac{b(u)}{\sigma^{2}(u)} \mathrm{d} u\right)
$$

and the scale function by $S(x)=\int_{0}^{x} s(t) \mathrm{d} t . X$ is recurrent if and only if $\lim _{x \rightarrow \pm \infty} S(x)= \pm \infty$. In the case of recurrence the diffusion admits a unique up to a constant multiple invariant measure $m(\mathrm{~d} x)$, given by $m(\mathrm{~d} x)=1 /\left(s(x) \sigma^{2}(x)\right) \mathrm{d} x$. Denote $M=\int_{-\infty}^{+\infty} m(\mathrm{~d} x)$. The diffusion is positively recurrent if and only if $M<\infty$. In this case put

$$
\mu(\mathrm{d} x)=p(x) \mathrm{d} x, \text { where } p(x)=\frac{1}{M s(x) \sigma^{2}(x)} .
$$

The probability $\mu$ is called invariant or stationary probability of $X$.

Using Assumptions 2.1.2 and 2.2.1, 2.2.2 we see that for any $x$ such that $|x| \leq M_{0}$,

$$
s^{-1}(x) \leq \mathrm{e}^{\frac{2 M_{0} b_{0}}{\sigma_{0}^{2}}}
$$

and for $|x| \geq M_{0}$,

$$
s^{-1}(x) \leq \mathrm{e}^{\frac{2 M_{0} b_{0}}{\sigma_{0}^{2}}}\left(\frac{M_{0}}{|x|}\right)^{\frac{2 \gamma}{\sigma_{1}^{2}}} .
$$

This shows that $S(x) \rightarrow \pm \infty$, when $x \rightarrow \pm \infty$. Hence $X$ is recurrent. The same estimation gives $M<\infty$ (and $X$ is positively recurrent) as soon as $2 \gamma>\sigma_{1}^{2}$.

Actually Assumption 2.2.3: $2 \gamma>31 \sigma_{1}^{2}$ guarantees more than positive recurrence. It is well known that the positive recurrence of $X$ is equivalent to $\mathbb{E}_{x} T_{a}<\infty$ for all $a \in \mathbb{R}, x \in \mathbb{R}$, where $T_{a}$ is the hitting time of level $a$. Under Assumptions 2.1.2 and 2.2.1, 2.2.2 the moments of hitting times of $X$ satisfy $\mathbb{E}_{x} T_{a}^{n}<\infty$ for $n<\gamma / \sigma_{1}^{2}+1 / 2$, for all $x \in \mathbb{R}, a \in \mathbb{R}$, see Loukianova et al. [16], Theorem 5.5. Thus under Assumption 2.2.3 we have $\mathbb{E}_{x} T_{a}^{n}<\infty$ for $n \leq 16$. This means that the "speed of recurrence" of $X$ is polynomial of order 16 and will be used to bound the speed of convergence of our estimator. Though we do not use the mixing coefficient, note that Assumption 2.2 guarantees that the diffusion is polynomially $\beta$-mixing (see Veretennikov [21]).

It follows from the above assumptions that the invariant density $p$ is continuous and hence bounded from above and below on any compact interval. So we have

$$
0<p_{0} \leq p(x) \leq p_{1}<\infty \text { for all } x \in[0,1] .
$$

In the sequel we need to fix $p_{0}$. We get immediately that

$$
M=\int_{-\infty}^{+\infty}\left(s(x) \sigma^{2}(x)\right)^{-1} \mathrm{~d} x \leq \frac{2 M_{0}}{\sigma_{0}^{2}} \mathrm{e}^{\frac{2 M_{0} b_{0}}{\sigma_{0}^{2}}}\left[\frac{2 \gamma}{2 \gamma-\sigma_{1}^{2}}\right]=: M_{+} .
$$


This yields the following lower bound for all $x \in[0,1]$

$$
p(x) \geq \frac{1}{M_{+}} \frac{1}{\sigma_{1}^{2}} \mathrm{e}^{-2 b_{0} / \sigma_{0}^{2}}:=p_{0} .
$$

In conclusion of this subsection, let us give an example of a diffusion process which fulfills Assumptions 2.2. Consider the solution of

$$
\mathrm{d} X_{t}=-\frac{\gamma X_{t}}{1+X_{t}^{2}} \mathrm{~d} t+\mathrm{d} W_{t}, \quad X_{0}=x, \quad \gamma>\frac{31}{2} .
$$

It is positive recurrent with stationary distribution

$$
\mu(\mathrm{d} x) \sim \frac{\mathrm{d} x}{\left(1+x^{2}\right)^{\gamma}}
$$

and satisfies all the assumptions of 2.2. Remark that there is no evidence whether this diffusion is exponentially $\beta$-mixing.

\subsection{Construction of the estimator}

In this section we introduce a nonparametric estimator of the unknown drift function $b$ on an interval $K$. We use the penalized least-squares based approach, where an estimator is constructed as a "projection" on some finite dimensional approximation space. We firstly address the non-adaptive case, where the statistician chooses himself the dimension of the approximation space. This choice can be done in an optimal way for example if the smoothness of the unknown function $b$ is known. Secondly we address the adaptive estimation procedure. In this case the dimension of the approximation space is chosen automatically using some penalization procedure, based on the data.

Consider a collection $\left\{\mathcal{S}_{m} ; m \in \mathcal{M}_{t}\right\}$ of approximation spaces. Each of these spaces is a linear finite dimensional subspace of $L^{2}(K, \mathrm{~d} x)$. Here $\mathcal{M}_{t}$ is a set of indices. We suppose that there exists a space denoted by $\mathcal{S}_{t}$, belonging to the collection, such that $\mathcal{S}_{m} \subseteq \mathcal{S}_{t}$ for all $m \in \mathcal{M}_{t}$. Denote by $D_{m}$ the dimension of $\mathcal{S}_{m}$ and by $D_{t}$ the dimension of $\mathcal{S}_{t}$.

Put

$$
\|h\|_{t}^{2}=\frac{1}{t} \int_{0}^{t} h^{2}\left(X_{s}\right) \mathrm{d} s
$$

and denote the corresponding quadratic form by

$$
T_{X}(h, f)=\frac{1}{t} \int_{0}^{t} h\left(X_{s}\right) f\left(X_{s}\right) \mathrm{d} s \text { for all } f, h \in \mathcal{S}_{t} .
$$

We firstly construct the non-adaptive estimator. To this end fix a linear subspace $\mathcal{S}_{m} \subset \mathcal{S}_{t}$. We shall write shortly $b_{K}(x):=b(x) 1_{K}(x)$ for the restriction of the function $b$ to the interval $K$. The estimator $\hat{b}_{m}$ of $b_{K}$ will be defined as trajectorial minimizer on $\mathcal{S}_{m}$ of the following contrast function:

$$
\gamma_{t}(h)=\|h\|_{t}^{2}-\frac{2}{t} \int_{0}^{t} h\left(X_{s}\right) \mathrm{d} X_{s} .
$$

To insure the existence of $\hat{b}_{m}$ we impose some condition under which $T_{X}$ is a.s. positive-definite on $\mathcal{S}_{t}$ and hence on each $\mathcal{S}_{m}, m \in \mathcal{M}_{t}$. Denote by $\|h\|$ the $L^{2}(K, \mathrm{~d} x)$-norm, and let

$$
\rho_{t}(X)=\inf _{h \in \mathcal{S}_{t} ;\|h\|=1} T_{X}(h, h)
$$

Put

$$
A_{t}=\left\{\rho_{t}(X) \geq t^{-1 / 2}\right\}
$$


Note that, since $\mathcal{S}_{t}$ is finite-dimensional, $\gamma_{t}$ is almost surely defined for all $h \in \mathcal{S}_{t}$ (see Rem. 2.3 below). We finally put

$$
\hat{b}_{m}=\arg \min _{h \in \mathcal{S}_{m}} \gamma_{t}(h) \text { on } A_{t} \text { and } \hat{b}_{m}=0 \text { on } A_{t}^{c} .
$$

Clearly, for all $\omega \in A_{t}, T_{X}$ is a strictly positive-definite quadratic form on $\mathcal{S}_{m}, m \in \mathcal{M}_{t}$, and $\gamma_{t}$ is a difference between this strictly positive quadratic form and a linear form. Hence the minimizer of $\gamma_{t}$ exists and is unique on $\mathcal{S}_{m}, m \in \mathcal{M}_{t}$. As it was explained, in the non-adaptive case the statistician chooses himself the approximation space.

In the adaptive case the dimension is chosen automatically using a model selection procedure. In order to describe this procedure, we have to define properly $\gamma_{t}\left(\hat{b}_{m}\right)$. Fix some basis $\left\{\varphi_{1}, \ldots, \varphi_{D_{m}}\right\}$ of $\mathcal{S}_{m}$. From the definition of $\gamma_{t}$ it follows that on $A_{t}$,

$$
\hat{b}_{m}=\sum_{i=1}^{D_{m}} \hat{\alpha}_{i} \varphi_{i}
$$

with random $\hat{\alpha}=\left(\hat{\alpha}_{1}, \ldots, \hat{\alpha}_{D_{m}}\right)^{*}$ (we denote by $*$ the usual matrix transposition) satisfying

$$
T^{\varphi} \hat{\alpha}=\frac{1}{t} \int_{0}^{t} \varphi\left(X_{s}\right) \mathrm{d} X_{s},
$$

where $T^{\varphi}$ is the $D_{m} \times D_{m}$ random matrix with elements

$$
T_{i j}^{\varphi}=\frac{1}{t} \int_{0}^{t} \varphi_{i}\left(X_{s}\right) \varphi_{j}\left(X_{s}\right) \mathrm{d} s
$$

and where

Define on $A_{t}$

$$
\int_{0}^{t} \varphi\left(X_{s}\right) \mathrm{d} X_{s}=\left(\begin{array}{c}
\int_{0}^{t} \varphi_{1}\left(X_{s}\right) \mathrm{d} X_{s} \\
\vdots \\
\int_{0}^{t} \varphi_{D_{m}}\left(X_{s}\right) \mathrm{d} X_{s}
\end{array}\right)
$$

$$
\gamma_{t}\left(\hat{b}_{m}\right):=\left\|\hat{b}_{m}\right\|_{t}^{2}-\frac{2}{t} \sum_{i=1}^{D_{m}} \hat{\alpha}_{i} \int_{0}^{t} \varphi_{i}\left(X_{s}\right) \mathrm{d} X_{s} .
$$

Now we are able to introduce the adaptive estimator. Define

$$
\hat{m}:=\arg \min _{m \in \mathcal{M}_{t}}\left[\gamma_{t}\left(\hat{b}_{m}\right)+\operatorname{pen}(m)\right]
$$

where the penalization term pen $(m)$ will be given later, see (2.7). Then the estimator that we propose is the following adaptive estimator

$$
\hat{b}_{\hat{m}}:= \begin{cases}\sum_{n} 1_{\{\hat{m}=n\}} \hat{b}_{n} & \text { on } A_{t} . \\ 0 & \text { on } A_{t}^{c}\end{cases}
$$

Remark 2.3. The above considerations and in particular the definition of $\gamma_{t}\left(\hat{b}_{m}\right)$ of $(2.5)$ do not depend on the special choice of bases.

Indeed, let $\left\{\varphi_{1}, \ldots, \varphi_{n}\right\}$ and $\left\{\psi_{1}, \ldots, \psi_{n}\right\}$ be two bases of $\mathcal{S}_{t}\left(\right.$ or $\left.\mathcal{S}_{m}\right)$, with $n=D_{t}$ (resp. $\left.D_{m}\right)$, and let $A=\left(a_{i j}\right)$ be the $n \times n$ matrix such that $\varphi_{i}=\sum_{j} a_{i j} \psi_{j}$, for any $1 \leq i \leq n$. We then have for a function $h$

$$
h=\sum_{i=1}^{n} \alpha_{i} \varphi_{i}=\sum_{i=1}^{n} \beta_{i} \psi_{i}
$$

where $\beta=A^{*} \alpha$. 
1. Hence, given a version of the stochastic integrals $\int \varphi_{i}\left(X_{s}\right) \mathrm{d} X_{s}, 1 \leq i \leq D_{t}$, the equalities

$$
\begin{aligned}
\int_{0}^{t} h\left(X_{s}\right) \mathrm{d} X_{s} & =\sum_{i=1}^{D_{t}} \alpha_{i} \int_{0}^{t} \varphi_{i}\left(X_{s}\right) \mathrm{d} X_{s}=\alpha^{*} \int_{0}^{t} \varphi\left(X_{s}\right) \mathrm{d} X_{s} \\
& =\alpha^{*} \int_{0}^{t} A \psi\left(X_{s}\right) \mathrm{d} X_{s}=\alpha^{*} A \int_{0}^{t} \psi\left(X_{s}\right) \mathrm{d} X_{s}=\sum_{i=1}^{D_{t}} \beta_{i} \int_{0}^{t} \psi_{i}\left(X_{s}\right) \mathrm{d} X_{s}
\end{aligned}
$$

determine automatically a version of any stochastic integral $\int h\left(X_{s}\right) \mathrm{d} X_{s}$ on $\mathcal{S}_{t}$, that does not depend on the choice of the basis.

2. From the definition $(2.5)$ of $\gamma_{t}\left(\hat{b}_{m}\right)$, we have

$$
\begin{aligned}
\gamma_{t}\left(\hat{b}_{m}\right) & =\left\|\hat{b}_{m}\right\|_{t}^{2}-\frac{2}{t}\left(\hat{\alpha}^{*} \int_{0}^{t} \varphi\left(X_{s}\right) \mathrm{d} X_{s}\right) \\
& =\left\|\hat{b}_{m}\right\|_{t}^{2}-\frac{2}{t}\left(\hat{\alpha}^{*} \int_{0}^{t} A \psi\left(X_{s}\right) \mathrm{d} X_{s}\right) \\
& =\left\|\hat{b}_{m}\right\|_{t}^{2}-\frac{2}{t}\left(\hat{\alpha}^{*} A \int_{0}^{t} \psi\left(X_{s}\right) \mathrm{d} X_{s}\right) \\
& =\left\|\hat{b}_{m}\right\|_{t}^{2}-\frac{2}{t}\left(\hat{\beta}^{*} \int_{0}^{t} \psi\left(X_{s}\right) \mathrm{d} X_{s}\right)
\end{aligned}
$$

where $\hat{\beta}=A^{*} \hat{\alpha}$. The equality (2.4) yields

$$
T^{\psi} \hat{\beta}=A^{-1} T^{\varphi}\left(A^{-1}\right)^{*} A^{*} \hat{\alpha}=\frac{1}{t} \int_{0}^{t} \psi\left(X_{s}\right) \mathrm{d} X_{s},
$$

hence $\hat{\beta}$ satisfies (2.4), when replacing all $\varphi_{i}$ by $\psi_{i}$. This implies that the definition of $\hat{b}_{m}$ and of $\gamma_{t}\left(\hat{b}_{m}\right)$ does not depend on the choice of a basis in $\mathcal{S}_{m}$.

\subsection{Assumptions on linear subspaces of $L^{2}(K, \mathrm{~d} x)$}

We assume that the approximation spaces satisfy the following conditions:

\section{Assumption 2.4.}

1. We suppose that there exists $\Phi_{0}>0$ such that for all $m \in \mathcal{M}_{t}$, for all $h \in \mathcal{S}_{m}$,

$$
\|h\|_{\infty} \leq \Phi_{0} D_{m}^{1 / 2}\|h\| .
$$

Recall that $\|h\|^{2}=\int_{K} h^{2}(x) \mathrm{d} x$ is the usual $L^{2}(K, \mathrm{~d} x)-$ norm.

2. We suppose that

where the constant $C$ does not depend on $t$.

$$
\sum_{m \in \mathcal{M}_{t}} \mathrm{e}^{-D_{m}} \leq C
$$

3. Dimension condition.

$$
D_{t} \leq t .
$$

4. We suppose that there exists an orthonormal basis $\left\{\varphi_{1}, \ldots, \varphi_{D_{t}}\right\}$ of $\mathcal{S}_{t} \subset L^{2}(K, d x)$ and a positive constant $\Phi_{1}$ such that for all $i$,

$$
\operatorname{card}\left\{j:\left\|\varphi_{i} \varphi_{j}\right\|_{\infty} \neq 0\right\} \leq \Phi_{1} .
$$


5. We suppose that the cardinality of $\mathcal{M}_{t}$ satisfies

$$
\operatorname{card} \mathcal{M}_{t} \leq D_{t}
$$

\subsection{Example for approximation spaces}

We present a collection of models that can be used for estimation. We consider the space of piecewise polynomials, as introduced for example in Baraud et al. [2,3] and Comte et al. [5].

Take $K=[0,1]$ and fix an integer $r \geq 0$. For $p \in \mathbb{N}$, consider the dyadic subintervals $I_{j, p}=\left[(j-1) 2^{-p}, j 2^{-p}\right]$, for any $1 \leq j \leq 2^{p}$. On each subinterval $I_{j, p}$, we consider polynomials of degree less or equal to $r$, so we have polynomials $\varphi_{j, l}, 0 \leq l \leq r$ of degree $l$, such that $\varphi_{j, l}$ is zero outside $I_{j, p}$. Then the space $\mathcal{S}_{m}$, for $m=(r, p)$, is defined as the space of all functions that can be written as

$$
t(x)=\sum_{j=1}^{2^{p}} \sum_{l=0}^{r} t_{j, l} \varphi_{j, l}(x) .
$$

Hence, $D_{m}=(r+1) 2^{p}$. Then the collection of spaces $\left\{\mathcal{S}_{m}, m \in \mathcal{M}_{t}\right\}$ is such that

$$
\mathcal{M}_{t}=\left\{m=(r, p), p \geq 0, r \in\left\{0, \ldots, r_{\max }\right\}, 2^{p}\left(r_{\max }+1\right) \leq D_{t}\right\}
$$

One possible choice of $\mathcal{S}_{t}$ and $D_{t}$ is as follows: take

$$
p_{\max }:=\max \left\{p: 2^{p}\left(r_{\max }+1\right) \leq t\right\}, D_{t}=2^{p_{\max }}\left(r_{\max }+1\right)
$$

and let $\mathcal{S}_{t}$ be the space of piecewise polynomials associated to $m_{\max }:=\left(r_{\max }, p_{\max }\right)$. Then it is evident that any of the spaces $\mathcal{S}_{m}, m \in \mathcal{M}_{t}$, is contained in $\mathcal{S}_{t}$. Furthermore, card $\mathcal{M}_{t}=\left(p_{\max }+1\right)\left(r_{\max }+1\right) \leq D_{t} \leq t$.

It is well known, see for instance Comte et al. [5], that for this model the assumption of norm connection 2.4.2.4 is satisfied. Note moreover that for a fixed $\varphi_{j, l} \in \mathcal{S}_{t}$,

$$
\operatorname{card}\left\{\left(j^{\prime}, l^{\prime}\right): \varphi_{j^{\prime}, l^{\prime}} \varphi_{j, l} \neq 0\right\}=\operatorname{card}\left\{\left(j, l^{\prime}\right): \varphi_{j, l^{\prime}} \varphi_{j, l} \neq 0\right\} \leq r_{\max }+1
$$

which does not depend on $t$. Hence assumption 2.4.2.4 is satisfied. Finally, it is easy to check that also Assumption 2.4.2.4 holds:

$$
\begin{aligned}
\sum_{m \in \mathcal{M}_{t}} \mathrm{e}^{-D_{m}} & =\sum_{r=0}^{r_{\max }} \sum_{p: 2^{p}\left(r_{\max }+1\right) \leq D_{t}} \mathrm{e}^{-(r+1) 2^{p}} \\
& \leq \sum_{r=0}^{r_{\max }} \sum_{p: 2^{p}\left(r_{\max }+1\right) \leq D_{t}} \mathrm{e}^{-2^{p}} \\
& \leq\left(r_{\max }+1\right) \sum_{k \geq 0} \mathrm{e}^{-k}<+\infty
\end{aligned}
$$

where the last quantity does not depend on $t$.

Spaces generated by compactly supported wavelets, similar to those considered by Hoffmann [10] and Baraud et al. [2] or [3] are also covered by Assumption 2.4. On the other hand, spaces spanned by the trigonometric basis do not fulfill Assumption 2.4.2.4 and therefore do not fit to our set-up. 


\subsection{Main results}

We have the following first result concerning the non-adaptive estimator. Recall that $b_{K}(x)=b(x) 1_{K}(x)$ is the restriction of the function $b$ to the interval $K$. We define the risk of the estimator $\hat{b}_{m}$ as

$$
\mathbb{E}_{x}\left\|\hat{b}_{m}-b_{K}\right\|_{t}^{2}=\mathbb{E}_{x}\left(\frac{1}{t} \int_{0}^{t}\left(\hat{b}_{m}-b_{K}\right)^{2}\left(X_{s}\right) \mathrm{d} s\right) .
$$

Let $b_{m}$ be the $L^{2}(K, \mathrm{~d} x)$-projection of $b_{K}$ onto $\mathcal{S}_{m}$. Then the following holds.

Theorem 2.5. Suppose that $t \geq t_{0}:=4 / p_{0}^{2}$. Suppose that $X$ satisfies Assumptions 2.1 and 2.2. Suppose that the collection of the approximation spaces satisfies Assumptions 2.4.2.4, 2.4.3-5. Then

$$
\mathbb{E}_{x}\left\|\hat{b}_{m}-b_{K}\right\|_{t}^{2} \leq 3 \kappa\left\|b_{m}-b_{K}\right\|^{2}+16 \sigma_{1}^{2} \frac{\kappa}{p_{0}} \frac{D_{m}}{t}+C t^{-1}
$$

Here, $\kappa=\kappa(t)=\frac{2}{\sigma_{0}^{2}}\left(\frac{2 \operatorname{diam}(K)}{t}+\frac{2 \sigma_{1}}{\sqrt{t}}+2 b_{0}+\frac{\sigma_{1}^{2}}{2}\right)$ (see Prop. 3.1), and $C$ is a positive constant depending on $b_{0}, \sigma_{1}$ and $\Phi_{0}$.

Let us give some comments on (2.6). It is natural to choose the dimension $D_{m}$ that balances the bias term $\left\|b_{m}-b_{K}\right\|^{2}$ and the variance term which is of order $D_{m} / t$. Assume that $b_{K}$ belongs to some Besov space $B_{2, \infty}^{\alpha}([0,1])$ and consider the space of piecewise polynomials $\mathcal{S}_{m}$ such that $r>\alpha-1$. Then it can be shown that $\left\|b_{m}-b_{K}\right\|^{2} \leq C D_{m}^{-2 \alpha}$, see for example Barron et al. [4], Lemma 12. Thus the best choice of $D_{m}$ is to take

$$
D_{m}=t^{\frac{1}{2 \alpha+1}}
$$

and then we obtain

$$
\mathbb{E}_{x}\left(\left\|\hat{b}_{m}-b_{K}\right\|_{t}^{2}\right) \leq C t^{-\frac{2 \alpha}{2 \alpha+1}}+C_{1} t^{-1}
$$

and this yields exactly the classical nonparametric rate $t^{-\frac{2 \alpha}{2 \alpha+1}}$ (compare for example to Hoffmann [10]). This choice however supposes the knowledge of the regularity $\alpha$ of the unknown drift function, and that is why an adaptive estimation scheme has to be used, in order to choose automatically the best dimension $D_{m}$ in the case when the regularity $\alpha$ is not known.

Concerning the adaptive drift estimator, we have the following theorem.

Theorem 2.6. Suppose that $X$ satisfies Assumptions 2.1 and 2.2. Suppose that the collection of the approximation spaces satisfies Assumption 2.4. Suppose that $t \geq t_{0}$, where $t_{0}:=4 / p_{0}^{2}$. Let

$$
\operatorname{pen}(m)=\chi \sigma_{1}^{2} \frac{D_{m}}{t}
$$

where $\chi$ is a universal constant that will be given explicitly in (4.11). Then we have

$$
\mathbb{E}_{x}\left\|\hat{b}_{\hat{m}}-b_{K}\right\|_{t}^{2} \leq 3 \kappa \inf _{m \in \mathcal{M}_{t}}\left(\left\|b_{m}-b_{K}\right\|^{2}+\operatorname{pen}(m)\right)+\frac{C}{t}
$$

where $\kappa=\kappa(t)=\frac{2}{\sigma_{0}^{2}}\left(\frac{2 \operatorname{diam}(K)}{t}+\frac{2 \sigma_{1}}{\sqrt{t}}+2 b_{0}+\frac{\sigma_{1}^{2}}{2}\right)$ (compare to Prop. 3.1) and where $C$ is a positive constant not depending on $t$.

\section{Probabilistic tools And auxiliary Results}

In this section, we collect some probabilistic results and auxiliary lemmas that are needed for the proofs of the main results. 


\subsection{Probabilistic tools}

In what follows we often need to compare empirical and theoretical norms. One way of doing this is given by the next proposition.

Proposition 3.1. For any positive function $f$ having support on a compact interval $K$, we have

$$
\frac{1}{t} \mathbb{E}_{x} \int_{0}^{t} f\left(X_{s}\right) \mathrm{d} s \leq \kappa(t) \int_{K} f(x) \mathrm{d} x
$$

where $\kappa(t)=\frac{2}{\sigma_{0}^{2}}\left(\frac{2 \operatorname{diam}(K)}{t}+\frac{2 \sigma_{1}}{\sqrt{t}}+2 b_{0}+\frac{\sigma_{1}^{2}}{2}\right)$.

Proof. By the occupation time formula and since $f$ has support in $K$,

$$
\mathbb{E}_{x} \int_{0}^{t} f\left(X_{s}\right) \mathrm{d} s=\int_{K} f(y) \frac{2}{\sigma^{2}(y)} \mathbb{E}_{x} L_{t}^{y} \mathrm{~d} y .
$$

We will derive a bound on $\mathbb{E}_{x} L_{t}^{y}$ for $y \in K$. Let $y_{0}$ be the leftmost point of $K$. We have

$$
\mathbb{E}_{x} L_{t}^{y_{0}}-\mathbb{E}_{x}\left|L_{t}^{y}-L_{t}^{y_{0}}\right| \leq \mathbb{E}_{x} L_{t}^{y} \leq \mathbb{E}_{x} L_{t}^{y_{0}}+\mathbb{E}_{x}\left|L_{t}^{y}-L_{t}^{y_{0}}\right|
$$

and

$$
\left|L_{t}^{y}-L_{t}^{y_{0}}\right| \leq\left|y-y_{0}\right|+\left|\int_{0}^{t} \mathbf{1}_{\left\{y_{0}<X_{s}<y\right\}} \sigma\left(X_{s}\right) \mathrm{d} W_{s}\right|+\int_{0}^{t} \mathbf{1}_{\left\{X_{s} \in K\right\}}\left|b\left(X_{s}\right)\right| \mathrm{d} s .
$$

Taking expectation we obtain

$$
\mathbb{E}_{x} \int_{0}^{t} \mathbf{1}_{\left\{X_{s} \in K\right\}}\left|b\left(X_{s}\right)\right| \mathrm{d} s \leq t b_{0}
$$

and by norm inclusion and isometry,

$$
\begin{aligned}
\mathbb{E}_{x}\left|\int_{0}^{t} 1_{\left\{y_{0}<X_{s}<y\right\}} \sigma\left(X_{s}\right) \mathrm{d} W_{s}\right| & \leq\left(\mathbb{E}_{x}\left(\int_{0}^{t} \mathbf{1}_{\left\{y_{0}<X_{s}<y\right\}} \sigma\left(X_{s}\right) \mathrm{d} W_{s}\right)^{2}\right)^{1 / 2} \\
& \leq\left(\mathbb{E}_{x}\left(\int_{0}^{t} \boldsymbol{1}_{\left\{X_{s} \in K\right\}} \sigma^{2}\left(X_{s}\right) \mathrm{d} s\right)\right)^{1 / 2} \leq \sigma_{1} \sqrt{t}
\end{aligned}
$$

In conclusion,

$$
\mathbb{E}_{x} L_{t}^{y} \leq \mathbb{E}_{x} L_{t}^{y_{0}}+\operatorname{diam}(K)+\sigma_{1} \sqrt{t}+t b_{0}=C_{0}+L,
$$

where $L:=\operatorname{diam}(K)+\sigma_{1} \sqrt{t}+t b_{0}$ and $C_{0}=\mathbb{E}_{x} L_{t}^{y_{0}}$. We also have $C_{0}-L \leq \mathbb{E}_{x} L_{t}^{y}$, so

$$
t \geq \mathbb{E}_{x} \int_{0}^{t} 1_{K}\left(X_{s}\right) \mathrm{d} s=\int_{K} \frac{2 \mathbb{E}_{x} L_{t}^{y}}{\sigma^{2}(y)} \mathrm{d} y \geq \frac{2\left(C_{0}-L\right)}{\sigma_{1}^{2}},
$$

whence

and thus finally,

$$
C_{0} \leq L+\sigma_{1}^{2} t / 2
$$

This concludes the proof.

$$
\mathbb{E}_{x} L_{t}^{y} \leq 2 L+\sigma_{1}^{2} t / 2=2\left(\operatorname{diam}(K)+\sigma_{1} \sqrt{t}+t b_{0}\right)+\sigma_{1}^{2} t / 2
$$

Now we give a useful deviation inequality for the one-dimensional ergodic diffusion process $X$, which is an immediate consequence of deviation inequality obtained by Loukianova et al. [16]. For $f: \mathbb{R} \rightarrow \mathbb{R}$ denote as usually $\mu(f)=\int_{\mathbb{R}} f \mathrm{~d} \mu$. 
Theorem 3.2 (deviation inequality). Let $f$ be a measurable bounded function with compact support such that $\mu(f) \neq 0$. Suppose that $X$ satisfies Assumptions 2.1 and 2.2.1, 2.2.2. Then for all $n \in \mathbb{N}$ such that

$$
n<\frac{\gamma}{\sigma_{1}^{2}}+\frac{1}{2}
$$

and any $0<\varepsilon \leq 1$, we have the following polynomial bound

$$
\mathbb{P}_{x}\left(\left|\frac{1}{t} \int_{0}^{t} f\left(X_{s}\right) \mathrm{d} s-\mu(f)\right| \geq \varepsilon\right) \leq K(n) t^{-n / 2} \varepsilon^{-n} \mu(|f|)^{n},
$$

where $K(n)$ is positive and finite, depending on the coefficients of the diffusion and on $n$ but not depending on $f, t, \varepsilon$.

This theorem follows directly from Theorems 4.3 and 5.5 of [16].

Corollary 3.3. Under Assumption 2.2.3 the previous theorem is satisfied for all $n \leq 16$.

\subsection{Auxiliary results}

In what follows we also need to compare empirical and theoretical norms through the set

$$
\Omega_{t}=\left\{\forall h \in \mathcal{S}_{t}, \quad \frac{1}{2} \mu\left(h^{2}\right) \leq\|h\|_{t}^{2} \leq \frac{3}{2} \mu\left(h^{2}\right)\right\},
$$

where any $h \in \mathcal{S}_{t}$ is defined as 0 outside of $K$. Recall that $A_{t}$ is given by (2.3) and $p_{0}$ by $(2.2)$.

Proposition 3.4. For all $t \geq 4 / p_{0}^{2}$ it holds that $\Omega_{t} \subseteq A_{t}$.

Proof. Note that by the definition of $A_{t}$ and $\Omega_{t}$, under the assumption $t \geq 4 / p_{0}^{2}$, the inequality $\mu\left(h^{2}\right) / 2 \leq\|h\|_{t}^{2}$ implies $\|h\|_{t}^{2} \geq p_{0}\|h\|^{2} / 2 \geq t^{-1 / 2}$, so $\Omega_{t} \subseteq A_{t}$.

Proposition 3.5. Suppose that $X$ satisfies Assumptions 2.1, 2.2.1 and 2.2.2. Suppose that the collection of approximation spaces $\left\{\mathcal{S}_{m}, m \in \mathcal{M}_{t}\right\}$ satisfies Assumptions 2.4.2.4, 2.4.2.4. Then for all

$$
n<\frac{\gamma}{\sigma_{1}^{2}}+\frac{1}{2}
$$

and for all $x \in \mathbb{R}$ we have that

$$
\mathbb{P}_{x}\left(\Omega_{t}^{c}\right) \leq C t^{-\frac{1}{2}(n-2)},
$$

where $C$ depends on $n$, the constant $\Phi_{1}$ given in Assumption 2.4.2.4 and on the coefficients of $X$, but does not depend on $t$.

Proof. Recall that $\|f\|$ denotes the usual $L^{2}(K, \mathrm{~d} x)$-norm. For any function $f$, write

$$
Z_{t}(f):=\frac{1}{t} \int_{0}^{t} f\left(X_{s}\right) \mathrm{d} s-\mu(f)
$$

Since for $f$ supported by $K,\|f\|_{\mu}^{2}=1$ implies that $\|f\|^{2} \leq p_{0}^{-1}$, we have that

$$
\mathbb{P}_{x}\left(\Omega_{t}^{c}\right) \leq \mathbb{P}_{x}\left(\sup _{f \in \mathcal{S}_{t},\|f\| \leq 1}\left|Z_{t}\left(f^{2}\right)\right|>0,5 p_{0}\right)
$$


Let $\left\{\varphi_{1}, \ldots, \varphi_{D_{t}}\right\}$ be an orthonormal basis of $\mathcal{S}_{t} \subset L^{2}(K, d x)$, satisfying Assumption 2.4.2.4, and note that any function $f$ with $\|f\| \leq 1$ can be written as

$$
f=\sum_{i=1}^{D_{t}} a_{i} \varphi_{i} \text { with } \sum a_{i}^{2} \leq 1 .
$$

Therefore,

$$
\begin{aligned}
\mathbb{P}_{x}\left(\Omega_{t}^{c}\right) & \leq \mathbb{P}_{x}\left(\sup _{\|f\| \leq 1}\left|Z_{t}\left(f^{2}\right)\right|>0,5 p_{0}\right) \\
& \leq \mathbb{P}_{x}\left(\sup _{\sum a_{i}^{2} \leq 1} \sum_{i, j} a_{i} a_{j}\left|Z_{t}\left(\varphi_{i} \varphi_{j}\right)\right|>0,5 p_{0}\right)
\end{aligned}
$$

Write

and fix some positive number $\varepsilon$. On the set

$$
C_{i j}:=\mu\left(\mid \varphi_{i} \varphi_{j}\right) \mid
$$

$$
\left\{\left|Z_{t}\left(\varphi_{i} \varphi_{j}\right)\right| \leq C_{i j} \varepsilon, \forall i, j\right\}
$$

we have that

$$
\sup _{\sum a_{i}^{2} \leq 1} \sum a_{i} a_{j}\left|Z_{t}\left(\varphi_{i} \varphi_{j}\right)\right| \leq \varepsilon \varrho(C),
$$

where $\varrho(C)$ is the biggest eigenvalue of the matrix $C$. Then choosing $\varepsilon:=p_{0} /(4 \varrho(C))$, we conclude that

$$
\mathbb{P}_{x}\left(\Omega_{t}^{c}\right) \leq \mathbb{P}_{x}\left(\exists i, j:\left|Z_{t}\left(\varphi_{i} \varphi_{j}\right)\right|>C_{i j} \varepsilon\right)
$$

By Theorem 3.2, we have the upper bound

$$
\mathbb{P}_{x}\left(\left|Z_{t}\left(\varphi_{i} \varphi_{j}\right)\right|>C_{i j} \varepsilon\right) \leq K(n) \varrho(C)^{n} t^{-n / 2} .
$$

Note that due to Assumption 2.4.2.4 and since $\mu\left(\left|\varphi_{i} \varphi_{j}\right|\right) \leq p_{1}$, we have that

$$
\varrho(C) \leq \Phi_{1} p_{1}
$$

where the upper bound does not depend on $t$. Indeed, using that $2 u_{i} u_{j} \leq u_{i}^{2}+u_{j}^{2}$, we have that

$$
\begin{aligned}
\varrho(C) & \left.=\sup _{u \in \mathbb{R}^{D_{t}},\|u\| \leq 1}<C u, u\right\rangle=\sup _{u \in \mathbb{R}^{D_{t}},\|u\| \leq 1} \sum_{i, j} C_{i j} u_{i} u_{j} \\
& \leq \sup _{u \in \mathbb{R}^{D_{t},\|u\| \leq 1}} \sum_{i, j} C_{i j} u_{i}^{2} \\
& =\sup _{u \in \mathbb{R}^{D_{t}},\|u\| \leq 1} \sum_{i} u_{i}^{2} \sum_{j: \varphi_{i} \varphi_{j} \neq 0} \mu\left(\left|\varphi_{i} \varphi_{j}\right|\right) \\
& \leq \sup _{u \in \mathbb{R}^{D_{t}},\|u\| \leq 1} \sum_{i} u_{i}^{2} \Phi_{1} p_{1} \leq \Phi_{1} p_{1} .
\end{aligned}
$$

Using once more that

$$
\sum_{i} \sum_{j} 1_{\left\{\varphi_{i} \varphi_{j} \neq 0\right\}} \leq D_{t} \cdot \Phi_{1},
$$


due to Assumption 2.4.2.4 we conclude that

$$
\mathbb{P}_{x}\left(\Omega_{t}^{c}\right) \leq C D_{t} t^{-n / 2} \leq C t^{-(n / 2-1)},
$$

where $C=K(n) \Phi_{1}^{n+1} p_{1}^{n}$ depends on $n$ and coefficients of $X$, but does not depend on $t$.

\section{Proofs of the Main Results}

\subsection{Proof of Theorem 2.5}

The proof follows the lines of Comte et al. [5]. Recall that from the definition of $\gamma_{t}$ it follows that on $A_{t}$,

$$
\hat{b}_{m}=\sum_{i=1}^{D_{m}} \hat{\alpha}_{i} \varphi_{i}
$$

with random $\hat{\alpha}=\left(\hat{\alpha}_{1}, \ldots, \hat{\alpha}_{D_{m}}\right)^{*}$ satisfying

$$
T \hat{\alpha}=\frac{1}{t} \int_{0}^{t} \varphi\left(X_{s}\right) \mathrm{d} X_{s}
$$

where $T$ is the $D_{m} \times D_{m}$ random matrix and $\int_{0}^{t} \varphi\left(X_{s}\right) \mathrm{d} X_{s}$ is the $D_{m}$-dimensional random vector with elements

$$
T_{i j}=\frac{1}{t} \int_{0}^{t} \varphi_{i}\left(X_{s}\right) \varphi_{j}\left(X_{s}\right) \mathrm{d} s, \quad \int_{0}^{t} \varphi\left(X_{s}\right) \mathrm{d} X_{s}=\left(\begin{array}{c}
\int_{0}^{t} \varphi_{1}\left(X_{s}\right) \mathrm{d} X_{s} \\
\vdots \\
\int_{0}^{t} \varphi_{D_{m}}\left(X_{s}\right) \mathrm{d} X_{s}
\end{array}\right) .
$$

Observe that $\hat{b}_{m}$ is a $\mathcal{F}_{t}$-measurable random variable with values in $\mathcal{S}_{m}$. If for such a random variable

$$
h(\omega, x)=\sum_{i=1}^{D_{m}} \alpha_{i}(\omega) \varphi_{i}(x)
$$

we put

$$
\gamma_{t}(h)=\|h\|_{t}^{2}-\frac{2}{t} \sum_{i=1}^{D_{m}} \alpha_{i} \int_{0}^{t} \varphi_{i}\left(X_{s}\right) \mathrm{d} X_{s} .
$$

Then $\gamma_{t}(h)-\gamma_{t}\left(\hat{b}_{m}\right) \geq 0$ on $A_{t}$. This inequality is evidently valid for any basis of $\mathcal{S}_{m}$.

Finally, we define the risk of the estimator $\hat{b}_{m}$ as

$$
\mathbb{E}_{x}\left\|\hat{b}_{m}-b_{K}\right\|_{t}^{2}=\mathbb{E}_{x}\left(\frac{1}{t} \int_{0}^{t}\left(\hat{b}_{m}-b_{K}\right)^{2}\left(X_{s}\right) \mathrm{d} s\right) .
$$

Let $\Omega_{t}$ be given by (3.1) and $A_{t}$ given by (2.3). Recall that $\Omega_{t} \subseteq A_{t}$ (Prop. 3.4.)

Now write

$$
\mathbb{E}_{x}\left\|\hat{b}_{m}-b_{K}\right\|_{t}^{2}=\mathbb{E}_{x}\left\|\hat{b}_{m}-b_{K}\right\|_{t}^{2} \mathbf{1}_{\Omega_{t}}+\mathbb{E}_{x}\left\|\hat{b}_{m}-b_{K}\right\|_{t}^{2} \mathbf{1}_{\Omega_{t}^{c}} .
$$

We will treat separately the two terms on the right-hand side.

We start with the first one, recalling that $\Omega_{t}=\Omega_{t} \cap A_{t}$. In what follows it will be useful to use an orthonormal basis $\left\{\psi_{1}, \ldots, \psi_{D_{m}}\right\}$ of $\mathcal{S}_{m}$ viewed as a subspace of $L^{2}(K, \mathrm{~d} \mu)$. Hence, our estimator can be rewritten as

$$
\hat{b}_{m}=\sum_{i=1}^{D_{m}} \hat{\beta}_{i} \psi_{i}, \text { and } b_{m}=\sum_{i=1}^{D_{m}} \beta_{i} \psi_{i}
$$


Observe that a.s. on $A_{t}$

$$
\begin{aligned}
0 \leq \gamma_{t}\left(\hat{b}_{m}\right)-\gamma_{t}\left(b_{m}\right) & =\left\|\hat{b}_{m}\right\|_{t}^{2}-\left\|b_{m}\right\|_{t}^{2}-\frac{2}{t} \sum_{i=1}^{D_{m}}\left(\hat{\beta}_{i}-\beta_{i}\right) \int_{0}^{t} \psi_{i}\left(X_{s}\right)\left(b\left(X_{s}\right) \mathrm{d} s+\sigma\left(X_{s}\right) \mathrm{d} W_{s}\right) \\
& =T_{X}\left(\hat{b}_{m}-b_{m}, \hat{b}_{m}+b_{m}\right)-2 T_{X}\left(\hat{b}_{m}-b_{m}, b_{K}\right)-\frac{2}{t} \sum_{i=1}^{D_{m}}\left(\hat{\beta}_{i}-\beta_{i}\right) \int_{0}^{t} \psi_{i}\left(X_{s}\right) \sigma\left(X_{s}\right) \mathrm{d} W_{s} \\
& =\left\|\hat{b}_{m}-b_{K}\right\|_{t}^{2}-\left\|b_{m}-b_{K}\right\|_{t}^{2}-\frac{2}{t} \sum_{i=1}^{D_{m}}\left(\hat{\beta}_{i}-\beta_{i}\right) \int_{0}^{t} \psi_{i}\left(X_{s}\right) \sigma\left(X_{s}\right) \mathrm{d} W_{s}
\end{aligned}
$$

whence a.s. on $A_{t}$

$$
\left\|\hat{b}_{m}-b_{K}\right\|_{t}^{2} \leq\left\|b_{m}-b_{K}\right\|_{t}^{2}+2 \sum_{i=1}^{D_{m}}\left(\hat{\beta}_{i}-\beta_{i}\right)\left(\frac{1}{t} \int_{0}^{t} \psi_{i}\left(X_{s}\right) \sigma\left(X_{s}\right) \mathrm{d} W_{s}\right) .
$$

Remark that $\sum_{i=1}^{D_{m}}\left(\hat{\beta}_{i}-\beta_{i}\right)^{2}=\left\|\hat{b}_{m}-b_{m}\right\|_{\mu}^{2}$. Using Cauchy-Schwartz inequality we have

$$
\begin{aligned}
\left\|\hat{b}_{m}-b_{K}\right\|_{t}^{2} \mathbf{1}_{\Omega_{t}} \leq & \left\|b_{m}-b_{K}\right\|_{t}^{2} \mathbf{1}_{\Omega_{t}}+2 \sum_{i=1}^{D_{m}}\left(\hat{\beta}_{i}-\beta_{i}\right)\left(\frac{1}{t} \int_{0}^{t} \psi_{i}\left(X_{s}\right) \sigma\left(X_{s}\right) \mathrm{d} W_{s}\right) \mathbf{1}_{\Omega_{t}} \\
\leq & \left\|b_{m}-b_{K}\right\|_{t}^{2}+\frac{1}{8}\left\|\hat{b}_{m}-b_{m}\right\|_{\mu}^{2} \mathbf{1}_{\Omega_{t}} \\
& +8 \sum_{i=1}^{D_{m}}\left(\frac{1}{t} \int_{0}^{t} \psi_{i}\left(X_{s}\right) \sigma\left(X_{s}\right) \mathrm{d} W_{s}\right)^{2} .
\end{aligned}
$$

Then on $\Omega_{t}$

$$
\frac{1}{8}\left\|\hat{b}_{m}-b_{m}\right\|_{\mu}^{2} \mathbf{1}_{\Omega_{t}} \leq \frac{1}{2}\left(\left\|\hat{b}_{m}-b_{K}\right\|_{t}^{2}+\left\|b_{m}-b_{K}\right\|_{t}^{2}\right) \mathbf{1}_{\Omega_{t}} .
$$

Plugging this into (4.2) gives

$$
\left\|\hat{b}_{m}-b_{K}\right\|_{t}^{2} \mathbf{1}_{\Omega_{t}} \leq 3\left\|b_{m}-b_{K}\right\|_{t}^{2}+16 \sum_{i=1}^{D_{m}}\left(\frac{1}{t} \int_{0}^{t} \psi_{i}\left(X_{s}\right) \sigma\left(X_{s}\right) \mathrm{d} W_{s}\right)^{2} .
$$

We have

$$
\mathbb{E}_{x}\left\|\hat{b}_{m}-b_{K}\right\|_{t}^{2} \mathbf{1}_{\Omega_{t}} \leq \frac{3}{t} \mathbb{E}_{x} \int_{0}^{t}\left(b_{m}-b_{K}\right)^{2}\left(X_{s}\right) \mathrm{d} s+\frac{16 \sigma_{1}^{2}}{t^{2}} \sum_{i=1}^{D_{m}} \mathbb{E}_{x} \int_{0}^{t} \psi_{i}^{2}\left(X_{s}\right) \mathrm{d} s .
$$

Using Proposition 3.1, we can write for any positive function $f$ having support on $K$,

$$
\mathbb{E}_{x} \int_{0}^{t} f\left(X_{s}\right) \mathrm{d} s \leq \kappa t \int_{K} f \mathrm{~d} x
$$

where the constant $\kappa$ is explicitly given in Proposition 3.1 and does only depend on the model constants $b_{0}, \sigma_{0}, \sigma_{1}$. Using this estimation, we obtain the following bound for the integrated risk restricted on $\Omega_{t}$ :

$$
\mathbb{E}_{x}\left\|\hat{b}_{m}-b_{K}\right\|_{t}^{2} \mathbf{1}_{\Omega_{t}} \leq 3 \kappa\left\|b_{m}-b_{K}\right\|^{2}+16 \sigma_{1}^{2} \frac{\kappa}{p_{0}} \frac{D_{m}}{t} .
$$


We now consider the risk restricted on $\Omega_{t}^{c}$. Recall that $A_{t}^{c} \subseteq \Omega_{t}^{c}$ and that $\hat{b}_{m}=0$ on $A_{t}^{c}$, and write

$$
\left\|b_{K}-\hat{b}_{m}\right\|_{t}^{2} \mathbf{1}_{\Omega_{t}^{c}}=\left\|b_{K}-\hat{b}_{m}\right\|_{t}^{2} \mathbf{1}_{\Omega_{t}^{c} \cap A_{t}}+\left\|b_{K}\right\|_{t}^{2} \mathbf{1}_{A_{t}^{c}}
$$

Let $\tilde{b}_{m}$ be the almost surely defined on $\Omega_{t}^{c} \cap A_{t}$ orthogonal projection of $b_{K}$ onto $\mathcal{S}_{m}$ w.r.t. $\|\cdot\|_{t}$. We have

$$
\begin{aligned}
\left\|b_{K}-\hat{b}_{m}\right\|_{t}^{2} \mathbf{1}_{\Omega_{t}^{c} \cap A_{t}} & =\left\|b_{K}-\tilde{b}_{m}\right\|_{t}^{2} \mathbf{1}_{\Omega_{t}^{c} \cap A_{t}}+\left\|\tilde{b}_{m}-\hat{b}_{m}\right\|_{t}^{2} \mathbf{1}_{\Omega_{t}^{c} \cap A_{t}} \\
& \leq\left\|b_{K}\right\|_{t}^{2} \mathbf{1}_{\Omega_{t}^{c} \cap A_{t}}+\left\|\tilde{b}_{m}-\hat{b}_{m}\right\|_{t}^{2} \mathbf{1}_{\Omega_{t}^{c} \cap A_{t}}
\end{aligned}
$$

which, combined with (4.3), implies

$$
\left\|b_{K}-\hat{b}_{m}\right\|_{t}^{2} \mathbf{1}_{\Omega_{t}^{c}} \leq\left\|b_{K}\right\|_{t}^{2} \mathbf{1}_{\Omega_{t}^{c}}+\left\|\tilde{b}_{m}-\hat{b}_{m}\right\|_{t}^{2} \mathbf{1}_{\Omega_{t}^{c} \cap A_{t}}
$$

Our Assumption 2.1.1 on $b(x)$ yields

$$
\mathbb{E}_{x}\left\|b_{K}\right\|_{t}^{2} \mathbf{1}_{\Omega_{t}^{c}} \leq b_{0}^{2} \mathbb{P}_{x}\left(\Omega_{t}^{c}\right) .
$$

From the definition of $\tilde{b}_{m}$ it follows that $\tilde{b}_{m}=\sum_{i=1}^{D_{m}} \tilde{\alpha}_{i} \varphi_{i}$, with $\tilde{\alpha}$ satisfying

$$
T \tilde{\alpha}=\frac{1}{t} \int_{0}^{t} \varphi\left(X_{s}\right) b\left(X_{s}\right) \mathrm{d} s
$$

Recall that on $A_{t}, \hat{b}_{m}=\sum_{i=1}^{D_{m}} \hat{\alpha}_{i} \varphi_{i}$, with $\hat{\alpha}$ given by (2.4). Hence on $A_{t}$, we can write $\hat{\alpha}-\tilde{\alpha}=T^{-1} M_{t}$, where

$$
M_{t}=\frac{1}{t} \int_{0}^{t} \varphi\left(X_{s}\right) \sigma\left(X_{s}\right) \mathrm{d} W_{s}=\left(\begin{array}{c}
\frac{1}{t} \int_{0}^{t} \varphi_{1}\left(X_{s}\right) \sigma\left(X_{s}\right) \mathrm{d} W_{s} \\
\vdots \\
\frac{1}{t} \int_{0}^{t} \varphi_{D_{m}}\left(X_{s}\right) \sigma\left(X_{s}\right) \mathrm{d} W_{s}
\end{array}\right) .
$$

So on $A_{t}$ we have $\hat{b}_{m}-\tilde{b}_{m}=\varphi^{*}(\hat{\alpha}-\tilde{\alpha})=\varphi^{*} T^{-1} M_{t}$, where $\varphi^{*}=\left(\varphi_{1}, \ldots \varphi_{D_{m}}\right)$, and (we denote by $*$ the matrix-transposition operation),

$$
\left(\hat{b}_{m}-\tilde{b}_{m}\right)^{2}\left(X_{s}\right)=M_{t}^{*}\left(T^{*}\right)^{-1} \varphi \varphi^{*}\left(X_{s}\right) T^{-1} M_{t} .
$$

So,

$$
\begin{aligned}
\left\|\tilde{b}_{m}-\hat{b}_{m}\right\|_{t}^{2} & =\frac{1}{t} \int_{0}^{t}\left(\tilde{b}_{m}-\hat{b}_{m}\right)^{2}\left(X_{s}\right) \mathrm{d} s \\
& =M_{t}^{*}\left(T^{*}\right)^{-1} T T^{-1} M_{t}=M_{t}^{*}\left(T^{*}\right)^{-1} M_{t}=\left\langle T^{-1} M_{t}, M_{t}\right\rangle,
\end{aligned}
$$

which gives, by the definition of $A_{t}$,

$$
\left\|\tilde{b}_{m}-\hat{b}_{m}\right\|_{t}^{2} \mathbf{1}_{\Omega_{t}^{c} \cap A_{t}} \leq \frac{1}{t^{-1 / 2}}\left\|M_{t}\right\|^{2} \mathbf{1}_{\Omega_{t}^{c}}=t^{1 / 2} \sum_{i=1}^{D_{m}}\left(\frac{1}{t} \int_{0}^{t} \varphi_{i}\left(X_{s}\right) \sigma\left(X_{s}\right) \mathrm{d} W_{s}\right)^{2} \mathbf{1}_{\Omega_{t}^{c}} .
$$


Using Burkholder-Davis-Gundy inequalities and the hypothesis $\left\|\varphi_{i}^{2}\right\|_{\infty} \leq \Phi_{0}^{2} D_{m}$, it follows from (4.6),

$$
\begin{aligned}
\mathbb{E}_{x}\left\|\tilde{b}_{m}-\hat{b}_{m}\right\|_{t}^{2} \mathbf{1}_{\Omega_{t}^{c} \cap A_{t}} & \leq \frac{t^{1 / 2}}{t^{2}} \sum_{i=1}^{D_{m}} \mathbb{E}_{x}\left(\left(\int_{0}^{t} \varphi_{i}\left(X_{s}\right) \sigma\left(X_{s}\right) \mathrm{d} W_{s}\right)^{2} 1_{\Omega_{t}^{c}}\right) \\
& \leq t^{-3 / 2} \sum_{i=1}^{D_{m}} \sqrt{\mathbb{E}_{x}\left(\int_{0}^{t} \varphi_{i}\left(X_{s}\right) \sigma\left(X_{s}\right) \mathrm{d} W s\right)^{4} \mathbb{P}_{x}\left(\Omega_{t}^{c}\right)} \\
& \leq t^{-3 / 2} \sum_{i=1}^{D_{m}} \sqrt{C(4) \mathbb{E}_{x}\left(\int_{0}^{t} \varphi_{i}^{2}\left(X_{s}\right) \sigma^{2}\left(X_{s}\right) \mathrm{d} s\right)^{2} \mathbb{P}_{x}\left(\Omega_{t}^{c}\right)}
\end{aligned}
$$

Here, $C(4)$ is a Burkholder-Davis-Gundy constant. But

$$
\int_{0}^{t} \varphi_{i}^{2}\left(X_{s}\right) \sigma^{2}\left(X_{s}\right) \mathrm{d} s \leq \Phi_{0}^{2} D_{m} \sigma_{1}^{2} t
$$

hence

$$
\begin{aligned}
\mathbb{E}_{x}\left\|\tilde{b}_{m}-\hat{b}_{m}\right\|_{t}^{2} \mathbf{1}_{\Omega_{t}^{c} \cap A_{t}} & \leq \sqrt{C(4)} t^{-3 / 2} \sum_{i=1}^{D_{m}} \sqrt{\Phi_{0}^{4} D_{m}^{2} \sigma_{1}^{4} t^{2} \mathbb{P}_{x}\left(\Omega_{t}^{c}\right)} \\
& \leq \sqrt{C(4)} \sigma_{1}^{2} \Phi_{0}^{2} t^{-1 / 2} D_{m}^{2} \sqrt{\mathbb{P}_{x}\left(\Omega_{t}^{c}\right)}
\end{aligned}
$$

From (4.4) and (4.5) the integrated risk on $\Omega_{t}^{c}$ satisfies

$$
\begin{aligned}
\mathbb{E}_{x}\left\|b_{K}-\hat{b}_{m}\right\|_{t}^{2} \mathbf{1}_{\Omega_{t}^{c}} & \leq\left(b_{0}^{2}+C \sigma_{1}^{2} \Phi_{0}^{2} t^{-1 / 2} D_{m}^{2}\right) \sqrt{\mathbb{P}_{x}\left(\Omega_{t}^{c}\right)} \\
& \leq\left(b_{0}^{2}+C \sigma_{1}^{2} \Phi_{0}^{2}\right) t^{-1 / 2} D_{m}^{2} \sqrt{\mathbb{P}_{x}\left(\Omega_{t}^{c}\right)}
\end{aligned}
$$

As a consequence, since $D_{m}^{2} \leq t^{2}$, the full integrated risk satisfies

$$
\begin{aligned}
\mathbb{E}_{x}\left\|\hat{b}_{m}-b_{K}\right\|_{t}^{2} \leq & 3 \kappa\left\|b_{m}-b_{K}\right\|^{2}+16 \sigma_{1}^{2} \frac{\kappa}{p_{0}} \frac{D_{m}}{t} \\
& +\left(b_{0}^{2}+C \sigma_{1}^{2} \Phi_{0}^{2}\right) t^{3 / 2} \sqrt{\mathbb{P}_{x}\left(\Omega_{t}^{c}\right)} .
\end{aligned}
$$

Finally, Proposition 3.5, applied with $n=12$, yields

$$
\mathbb{P}_{x}\left(\Omega_{t}^{c}\right) \leq \frac{C}{t^{5}}
$$

for $t \geq t_{0}$. This finishes the proof.

Remark 4.1. In the case when $X$ is in the stationary regime, i.e. starting from the invariant measure $\mu$, (2.6) can be improved to

$$
\mathbb{E}_{\mu}\left\|\hat{b}_{m}-b_{K}\right\|_{t}^{2} \leq 3 p_{1}\left\|b_{m}-b_{K}\right\|^{2}+16 \sigma_{1}^{2} \frac{D_{m}}{t}+C t^{-1}
$$

\subsection{Proof of Theorem $\mathbf{2 . 6}$}

Put

$$
\nu_{t}(f):=\frac{1}{t} \int_{0}^{t} f\left(X_{s}\right) \sigma\left(X_{s}\right) \mathrm{d} W_{s}
$$


The same argument that yields (4.1) in the non-adaptive case gives for any $m \in \mathcal{M}_{t}$,

$$
\left\|\hat{b}_{\hat{m}}-b_{K}\right\|_{t}^{2} 1_{A_{t}} \leq\left\|b_{m}-b_{K}\right\|_{t}^{2} 1_{A_{t}}+2 \nu_{t}\left(\hat{b}_{\hat{m}}-b_{m}\right) 1_{A_{t}}+(\operatorname{pen}(m)-\operatorname{pen}(\hat{m})) 1_{A_{t}} .
$$

Here, a special attention has to be paid to the term $\nu_{t}\left(\hat{b}_{\hat{m}}-b_{m}\right)$, since it is not a priori clear that this stochastic integral is well-defined. On $\hat{m}=n, \hat{b}_{\hat{m}}-b_{m}$ is an element of $\mathcal{S}_{n}+\mathcal{S}_{m}$ viewed as linear subspace of $L^{2}(K, \mu)$. Put $k=\operatorname{dim}\left(\mathcal{S}_{n}+\mathcal{S}_{m}\right)$ and let $\left\{\psi_{1}, \ldots, \psi_{k}\right\}$ be an orthonormal basis of this subspace. Then $1_{\{\hat{m}=n\}}\left(\hat{b}_{\hat{m}}-b_{m}\right)=1_{\{\hat{m}=n\}} \sum_{i=1}^{k} \hat{\beta}_{i} \psi_{i}$, and we define on $\hat{m}=n$,

$$
\nu_{t}\left(\hat{b}_{\hat{m}}-b_{m}\right):=\sum_{i=1}^{k} \hat{\beta}_{i} \nu_{t}\left(\psi_{i}\right)
$$

Hence, $\nu_{t}\left(\hat{b}_{\hat{m}}-b_{m}\right)$ is well-defined and linear. Thus we may write

$$
\nu_{t}\left(\hat{b}_{\hat{m}}-b_{m}\right) \leq\left\|\hat{b}_{\hat{m}}-b_{m}\right\|_{\mu} \cdot \nu_{t}\left(\frac{\hat{b}_{\hat{m}}-b_{m}}{\left\|\hat{b}_{\hat{m}}-b_{m}\right\|_{\mu}}\right) \leq\left\|\hat{b}_{\hat{m}}-b_{m}\right\|_{\mu} \cdot \sup _{h \in \mathcal{S}_{m}+\mathcal{S}_{\hat{m}},\|h\|_{\mu}=1}\left|\nu_{t}(h)\right| .
$$

Write for short

$$
G_{m}\left(m^{\prime}\right):=\sup _{h \in \mathcal{S}_{m}+\mathcal{S}_{m^{\prime}},\|h\|_{\mu}=1}\left|\nu_{t}(h)\right| .
$$

We now investigate (4.8). First, on $A_{t} \cap \Omega_{t}$, using that $2 a b \leq \frac{1}{8} a^{2}+8 b^{2}$,

$$
\begin{aligned}
\left\|\hat{b}_{\hat{m}}-b_{K}\right\|_{t}^{2} \leq & \left\|b_{m}-b_{K}\right\|_{t}^{2}+2\left\|\hat{b}_{\hat{m}}-b_{m}\right\|_{\mu} \quad G_{m}(\hat{m})+[\operatorname{pen}(m)-\operatorname{pen}(\hat{m})] \\
\leq & \left\|b_{m}-b_{K}\right\|_{t}^{2}+\frac{1}{8}\left\|\hat{b}_{\hat{m}}-b_{m}\right\|_{\mu}^{2}+8 G_{m}^{2}(\hat{m})+[\operatorname{pen}(m)-\operatorname{pen}(\hat{m})] \\
\leq & \left\|b_{m}-b_{K}\right\|_{t}^{2}+\frac{1}{2}\left(\left\|\hat{b}_{\hat{m}}-b_{K}\right\|_{t}^{2}+\left\|b_{K}-b_{m}\right\|_{t}^{2}\right) \\
& +8 G_{m}^{2}(\hat{m})+[\operatorname{pen}(m)-\operatorname{pen}(\hat{m})] \\
\leq & \frac{3}{2}\left\|b_{m}-b_{K}\right\|_{t}^{2}+\frac{1}{2}\left\|\hat{b}_{\hat{m}}-b_{K}\right\|_{t}^{2}+8 G_{m}^{2}(\hat{m})+[\operatorname{pen}(m)-\operatorname{pen}(\hat{m})] .
\end{aligned}
$$

This yields finally, on $A_{t} \cap \Omega_{t}=\Omega_{t}$,

$$
\left\|\hat{b}_{\hat{m}}-b_{K}\right\|_{t}^{2} \leq 3\left\|b_{m}-b_{K}\right\|_{t}^{2}+16 G_{m}^{2}(\hat{m})+2[\operatorname{pen}(m)-\operatorname{pen}(\hat{m})] .
$$

Now, as in Comte et al. [5], put $p\left(m, m^{\prime}\right):=p(m)+p\left(m^{\prime}\right)$, where

$$
p(m):=\chi \sigma_{1}^{2} \frac{D_{m}}{t}
$$

and where $\chi$ is a universal constant. Then

$$
\begin{aligned}
G_{m}^{2}(\hat{m}) 1_{\Omega_{t}} & \leq\left[\left(G_{m}^{2}(\hat{m})-p(m, \hat{m})\right) 1_{\Omega_{t}}\right]_{+}+p(m, \hat{m}) \\
& \leq \sum_{n \in \mathcal{M}_{t}}\left[\left(G_{m}^{2}(n)-p(m, n)\right) 1_{\Omega_{t}}\right]_{+}+p(m, \hat{m})
\end{aligned}
$$


Now we choose $\operatorname{pen}(m)$ such that $8 p\left(m, m^{\prime}\right) \leq \operatorname{pen}(m)+\operatorname{pen}\left(m^{\prime}\right)$, i.e.

$$
\operatorname{pen}(m):=8 \chi \sigma_{1}^{2} \frac{D_{m}}{t} .
$$

We have (see also Baraud et al. [3]):

Lemma 4.2. Under the assumptions of Theorem 2.6,

$$
\mathbb{E}_{x}\left(\left(G_{m}^{2}\left(m^{\prime}\right)-p\left(m, m^{\prime}\right)\right) 1_{\Omega_{t}}\right)_{+} \leq 1,6 \chi \sigma_{1}^{2} \frac{1}{t} \mathrm{e}^{-D_{m^{\prime}}}
$$

where $\chi$ is given by

$$
\chi=3 c\left(\delta_{0}\right)\left(1+2 \delta_{0}\right)\left(1+15 \delta_{0}\right), c\left(\delta_{0}\right)=\max \left(2 \ln 2+1, \ln \left(\frac{9}{2 \delta_{0}^{2}}\right)\right) .
$$

Here, $0<\delta_{0}<1$ is a free parameter. For the choice $\delta_{0}=0,0138$ this yields a constant $\chi=38$.

The proof of Lemma 4.2 will be given in Section 4.2 below.

For any $n$, let $\left\{\varphi_{1}^{n}, \ldots, \varphi_{D_{n}}^{n}\right\}$ be an orthonormal basis of $\mathcal{S}_{n}$. On $A_{t} \cap \Omega_{t}^{c}$, using (4.4) and (4.6), we have

$$
\begin{aligned}
\left\|\hat{b}_{\hat{m}}-b_{K}\right\|_{t}^{2} 1_{\left\{A_{t} \cap \Omega_{t}^{c}\right\}} & =\sum_{n \in \mathcal{M}_{t}} 1_{\left\{\hat{m}=n ; A_{t} \cap \Omega_{t}^{c}\right\}}\left\|\hat{b}_{n}-b_{K}\right\|_{t}^{2} \\
& \leq\left\|b_{K}\right\|_{t}^{2} \mathbf{1}_{\Omega_{t}^{c}}+\sum_{n \in \mathcal{M}_{t}} 1_{\{\hat{m}=n\}}\left\|\tilde{b}_{n}-\hat{b}_{n}\right\|_{t}^{2} \mathbf{1}_{\Omega_{t}^{c} \cap A_{t}} \\
& \leq\left\|b_{K}\right\|_{t}^{2} \mathbf{1}_{\Omega_{t}^{c}}+\sum_{n \in \mathcal{M}_{t}} 1_{\{\hat{m}=n\}} t^{1 / 2} \sum_{i=1}^{D_{n}}\left(\frac{1}{t} \int_{0}^{t} \varphi_{i}^{n}\left(X_{s}\right) \sigma\left(X_{s}\right) \mathrm{d} W_{s}\right)^{2} \mathbf{1}_{\Omega_{t}^{c}} \\
& \leq\left\|b_{K}\right\|_{t}^{2} \mathbf{1}_{\Omega_{t}^{c}}+\sum_{n \in \mathcal{M}_{t}} t^{1 / 2} \sum_{i=1}^{D_{n}}\left(\frac{1}{t} \int_{0}^{t} \varphi_{i}^{n}\left(X_{s}\right) \sigma\left(X_{s}\right) \mathrm{d} W_{s}\right)^{2} \mathbf{1}_{\Omega_{t}^{c}},
\end{aligned}
$$

The same calculus that yields (4.7) now gives

$$
\mathbb{E}_{x}|| \hat{b}_{\hat{m}}-b_{K} \|_{t}^{2} 1_{\left\{A_{t} \cap \Omega_{t}^{c}\right\}} \leq C\left(b_{0}^{2}+\sigma_{1}^{2} \Phi_{0}^{2}\right) t^{-1 / 2} D_{t}^{2}\left|\mathcal{M}_{t}\right| \sqrt{\mathbb{P}_{x}\left(\Omega_{t}^{c}\right)} .
$$

(4.9), (4.10) and (4.12) yield finally, for any $m$, using Assumption 2.4,

$$
\begin{aligned}
\mathbb{E}_{x}\left\|\hat{b}_{\hat{m}}-b_{K}\right\|_{t}^{2} 1_{A_{t}} \leq & 3 \mathbb{E}_{x}\left\|b_{m}-b_{K}\right\|_{t}^{2}+4 \operatorname{pen}(m)+16 \sum_{n \in \mathcal{M}_{t}} 1,6 \chi \sigma_{1}^{2} \frac{1}{t} \mathrm{e}^{-D_{n}} \\
& +C\left(b_{0}^{2}+\sigma_{1}^{2} \Phi_{0}^{2}\right) t^{-1 / 2} D_{t}^{2}\left|\mathcal{M}_{t}\right| \sqrt{\mathbb{P}_{x}\left(\Omega_{t}^{c}\right)} \\
\leq & 3 \kappa\left\|b_{m}-b\right\|^{2}+4 \operatorname{pen}(m)+C \chi \sigma_{1}^{2} \frac{1}{t} \\
& +C\left(b_{0}^{2}, \sigma_{1}^{2}\right) t^{-1 / 2} D_{t}^{3} \mathbb{P}_{x}\left(\Omega_{t}^{c}\right)^{1 / 2}
\end{aligned}
$$

Now, recall that by Proposition 3.5 , since $D_{t}^{3} \leq t^{3}$, taking $n=16$,

$$
\mathbb{P}_{x}\left(\Omega_{t}^{c}\right)^{1 / 2} \leq C t^{-7 / 2}
$$

and by Proposition 3.4,

$$
\mathbb{P}_{x}\left(A_{t}^{c}\right) \leq C t^{-1}
$$


As a consequence,

$$
\mathbb{E}_{x}\left\|\hat{b}_{\hat{m}}-b_{K}\right\|_{t}^{2} \leq 3 \kappa \inf _{m \in \mathcal{M}_{t}}\left(\left\|b_{m}-b\right\|^{2}+\operatorname{pen}(m)\right)+C \chi \sigma_{1}^{2} \frac{1}{t}+C\left(b_{0}^{2}, \sigma_{1}^{2}\right) t^{-1}
$$

This finishes the proof.

\section{A. Appendix}

In this appendix we give the proof of Lemma 4.2. Write

$$
\nu_{t}(f):=\frac{1}{t} \int_{0}^{t} f\left(X_{s}\right) \sigma\left(X_{s}\right) \mathrm{d} W_{s}
$$

Using the classical Bernstein inequality for continuous martingales, we recall that for all $a>0, v \neq 0$

$$
\mathbb{P}_{x}\left(\nu_{t}(f) \geq a,\|f\|_{t}^{2} \leq v^{2}\right) \leq \exp \left(-\frac{t a^{2}}{2 \sigma_{1}^{2} v^{2}}\right) .
$$

Recall that $\|f\|_{t}^{2}=\frac{1}{t} \int_{0}^{t} f^{2}\left(X_{s}\right) \mathrm{d} s$ and $\|f\|_{\mu}^{2}=\mu\left(f^{2}\right)$.

The proof of Proposition 4.2 follows Baraud et al. [3], pages 45-47. The chaining arguments which permits to state exponential bounds on supremum of empirical processes can also be found in Barron et al. [4]. By Lorentz et al. [13], for any linear subspace $S$ of $L^{2}([0,1], \mu)$ of dimension $d$, one can find a set $T_{\delta} \subset B$, where $B$ is the unit ball of $S \subset L^{2}([0,1], \mu)$, such that

$$
\operatorname{card}\left(T_{\delta}\right) \leq\left(\frac{3}{\delta}\right)^{d} \text {, and } \forall f \in B \exists f_{\delta} \in T_{\delta}:\left\|f-f_{\delta}\right\|_{\mu} \leq \delta \text {. }
$$

Apply this to the linear space $\mathcal{S}_{m}+\mathcal{S}_{m^{\prime}}$ of dimension $d\left(m^{\prime}\right) \leq D_{m}+D_{m^{\prime}}$. Consider $\delta_{k}-$ sets $T_{k}=T_{\delta_{k}}$ where $\delta_{k}=\delta_{0} 2^{-k}$, where $\delta_{0}<1$ is to be chosen later. Set $H_{k}:=\log \operatorname{card}\left(T_{k}\right)$. Write $B_{m^{\prime}}:=\left\{f \in \mathcal{S}_{m}+\mathcal{S}_{m^{\prime}}:\|f\|_{\mu} \leq 1\right\}$. Then for any $f \in B_{m^{\prime}}$, one can find a sequence $\left(f_{k}\right)_{k}$ with $f_{k} \in T_{k}$ such that $\left\|f-f_{k}\right\|_{\mu} \leq \delta_{k}$. Hence we get

$$
f=f_{0}+\sum_{k \geq 1}\left(f_{k}-f_{k-1}\right)
$$

Then as in Baraud et al. [3],

$$
\left\|f_{0}\right\|_{\mu} \leq 1,\left\|f_{k}-f_{k-1}\right\|_{\mu}^{2} \leq 5 \delta_{k-1}^{2} / 2
$$

In the following, we shall work in restriction to the set $\Omega_{t}$. Write $\mathbb{P}_{t}$ for the measure $\mathbb{P}_{x}\left(\cdot \cap \Omega_{t}\right)$. Put as in Baraud et al. [3],

$$
\Delta:=\sqrt{3} \sigma_{1}\left(\sqrt{x_{0}}+\sum_{k \geq 1} \delta_{k-1} \sqrt{5 x_{k} / 2}\right)
$$

then we have that

$$
\begin{aligned}
\mathbb{P}_{t}\left(\sup _{f \in B_{m^{\prime}}} \nu_{t}(f) \geq \Delta\right) & =\mathbb{P}_{t}\left(\exists\left(f_{k}\right)_{k}, f_{k} \in T_{k}: \nu_{t}\left(f_{0}\right)+\sum_{k \geq 1} \nu_{t}\left(f_{k}-f_{k-1}\right) \geq \Delta\right) \\
& \leq P_{1}+P_{2},
\end{aligned}
$$


where

$$
P_{1}=\sum_{f_{0} \in T_{0}} \mathbb{P}_{t}\left(\nu_{t}\left(f_{0}\right) \geq \sqrt{3 x_{0}} \sigma_{1}\right)
$$

and

$$
P_{2}=\sum_{k \geq 1} \sum_{f_{k} \in T_{k}, f_{k-1} \in T_{k-1}} \mathbb{P}_{t}\left(\nu_{t}\left(f_{k}-f_{k-1}\right) \geq \sigma_{1} \delta_{k-1} \sqrt{15 x_{k} / 2}\right) .
$$

Recall (4.13): Since on $\Omega_{t},\|f\|_{t}^{2} \leq \frac{3}{2}\|f\|_{\mu}^{2}$, we have for all $x>0$,

$$
\mathbb{P}_{t}\left(\nu_{t}(f) \geq \sqrt{3} \sigma_{1} \sqrt{x}\|f\|_{\mu}\right) \leq \exp (-t x) .
$$

We apply this inequality, remarking that $\left\|f_{0}\right\|_{\mu} \leq 1$, hence

$$
P_{1} \leq \operatorname{card}\left(T_{0}\right) \exp \left(-t x_{0}\right)=\exp \left(H_{0}-t x_{0}\right)
$$

and, since $\left\|f_{k}-f_{k-1}\right\|_{\mu}^{2} \leq 5 \delta_{k-1}^{2} / 2$,

$$
P_{2} \leq \sum_{k \geq 1} \exp \left(H_{k-1}+H_{k}-t x_{k}\right)
$$

Now, choose $x_{0}$ such that

$$
t x_{0}=H_{0}+D_{m^{\prime}}+\tau
$$

and $x_{k}$ such that

$$
t x_{k}=H_{k-1}+H_{k}+D_{m^{\prime}}+k d\left(m^{\prime}\right)+\tau .
$$

Then, if $d\left(m^{\prime}\right) \geq 1$, we obtain as in Baraud et al. [3],

$$
\mathbb{P}_{t}\left(\sup _{f \in B_{m^{\prime}}} \nu_{t}(f) \geq \Delta\right) \leq 1,6 \mathrm{e}^{-\tau} \mathrm{e}^{-D_{m^{\prime}}}
$$

Else, $d\left(m^{\prime}\right)=0$, hence $\mathcal{S}_{m}+\mathcal{S}_{m^{\prime}}=\{0\}$, and (4.14) holds trivially. Exactly as in Baraud et al. [3], it can be shown that

$$
t \Delta^{2} \leq \chi \sigma_{1}^{2}\left(D_{m^{\prime}}+D_{m}+\tau\right),
$$

where $\chi$ is the constant given in (4.11), and then we conclude as there

$$
\mathbb{E}_{x}\left[\left(G_{m}^{2}\left(m^{\prime}\right)-\chi \sigma_{1}^{2} \frac{D_{m^{\prime}}+D_{m}}{t}\right)_{+} 1_{\Omega_{t}}\right] \leq 1,6 \chi \sigma_{1}^{2} \frac{1}{t} \mathrm{e}^{-D_{m^{\prime}}}
$$

Acknowledgements. The subject of this paper has been proposed to the authors by Fabienne Comte and Valentine GenonCatalot during their research period at the University Paris-Descartes in spring 2008. The authors thank both of them for their kindness, their patience, and last, but not least for all the time spent on discussions and explanations on the paper. The authors are also grateful to the referees for comments that helped to significantly improve the paper. Eva Löcherbach has been partially supported by an ANR project: Ce travail a bénéficié d'une aide de l'Agence Nationale de la Recherche portant la référence ANR-08-BLAN-0220-01. 


\section{REFERENCES}

[1] G. Banon, Nonparametric identification for diffusion processes. SIAM J. Control. Optim. 16 (1978) 380-395.

[2] Y. Baraud, F. Comte and G. Viennet, Adaptive estimation in autoregression or $\beta$-mixing regression via model selection. Ann. Stat. 29 (2001) 839-875.

[3] Y. Baraud, F. Comte and G. Viennet, Model selection for (auto-)regression with dependent data. ESAIM: PESS 5 (2001) 33-49.

[4] A. Barron, L. Birgé and P. Massart, Risks bounds for model selection via penalization. Prob. Th. Rel. Fields 113 (1999) 301-413.

[5] F. Comte, V. Genon-Catalot and Y. Rozenholc, Penalized nonparametric mean square estimation of the coefficients of diffusion processes. Bernoulli 13 (2007) 514-543.

[6] A. Dalalyan, Sharp adaptive estimation of the drift function for ergodic diffusions. Ann. Stat. 33 (2005) 507-2528.

[7] A.S. Dalalyan and Yu.A. Kutoyants, Asymptotically efficient trend coefficient estimation for ergodic diffusion. Math. Meth. Stat. 11 (2002) 402-427.

[8] S. Delattre, M. Hoffmann and M. Kessler, Dynamics adaptive estimation of a scalar diffusion. Prpublication PMA-762, Univ. Paris 6 (2002). Available at www.proba.jussieu.fr/mathdoc/preprints/. Mathematical Reviews (MathSciNet): MR1895888 Project Euclid: euclid.bj/1078866865.

[9] L. Galtchouk and S. Pergamenschikov, Sequential nonparametric adaptive estimation of the drift coefficient in the diffusion processes. Math. Meth. Stat. 10 (2001) 316-330.

[10] M. Hoffmann, Adaptive estimation in diffusion processes. Stochastic Processes Appl. 79 (1999) 135-163.

[11] Yury A. Kutoyants, Statistical inference for ergodic diffusion processes. Springer Series in Statistics. London: Springer (2004).

[12] O. Lepskii, One problem of adaptive estimation in Gaussian white noise. Theory Probab. Appl. 35 (1999) 459-470.

[13] G.G. Lorentz, M. von Golitschek and Y. Makovoz, Constructive approximation: advanced problems. Grundlehren der Mathematischen Wissenschaften 304. Berlin: Springer (1996).

[14] D. Loukianova and O. Loukianov, Uniform deterministic equivalent of additive functionals and non-parametric drift estimation for one-dimensional recurrent diffusions. Ann. Inst. Henri Poincaré 44 (2008) 771-786.

[15] E. Löcherbach and D. Loukianova, On Nummelin splitting for continuous time Harris recurrent Markov processes and application to kernel estimation for multi-dimensional diffusions. Stoch. Proc. Appl. 118 (2008) 301-1321.

[16] E. Löcherbach, D. Loukianova and O. Loukianov, Polynomial bounds in the Ergodic theorem for one-dimensional diffusions and integrability of hitting times. Ann. Inst. H. Poincaré Probab. Statist. 47 (2011) 425-449.

[17] T.D. Pham, Nonparametric estimation of the drift coefficient in the diffusion equation. Math. Operationsforsch. Statist., Ser. Statistics 1 (1981) 61-73.

[18] B.L.S. Prakasa Rao, Statistical Inference for Diffusion Type Processes. London: Edward Arnold. MR1717690 (1999)

[19] D. Revuz and M. Yor, Continuous martingales and Brownian motion. 3rd ed. Grundlehren der Mathematischen Wissenschaften 293. Berlin: Springer (2005)

[20] V.G. Spokoiny, Adaptive drift estimation for nonparametric diffusion model. Ann. Stat. 28 (2000) 815-836.

[21] A.Yu. Veretennikov, On polynomial mixing bounds for stochastic differential equations. Stoch. Proc. Appl. 70 (1997) 115-127. 